\title{
Indikation und Hautqualität bestimmen die Produktwahl
}

Will man ästhetische Behandlungsergebnisse erzielen, die den individuellen Bedürfnissen der Patienten entsprechen, bedarf es einer breiten Produktpalette. Dabei sollte sich die Wahl des Fillers oder Skinboosters an Indikation und Hautqualität orientieren, berichteten Ästhetik-Spezialisten anlässlich der 25. Fortbildungswoche für praktische Dermatologie und Venerologie, München.

\section{Kombinationsbehandlung gewinnt an Bedeutung}

Die Kombination ästhetischer Behandlungsmethoden zur Faltenkorrektur, dem Volumenaufbau sowie der Verbesserung des Hautbildes gewinnt zunehmend an Bedeutung. Doch oftmals stelle sich die Frage „Wofür verwende ich was?“, sagte Dr. Anna Mokosch, Dermatologin aus Düsseldorf. Das Produktspektrum der Firma Galderma - bestehend aus Fillern und Skinboostern auf Hyaluronbasis und Botulinumtoxin - sei jetzt in vier
Kategorien unterteilt, die den Anwendern die Auswahl geeigneter Produkte und deren Kombinationen erleichtern. Die Bezeichnungen Relax, Restore, Enhance und Refresh orientiere sich an den Patientenbedürfnissen bzw. am gewünschten Behandlungsziel, erklärte Mokosch.

Der Einsatz von Botulinumtoxin Typ A (Azzalure ${ }^{\varpi}$, Relax) entspanne mimische Falten (z. B. Glabella), mit den Fillern der Restore-Reihe werde Volumen wieder aufgefüllt und altersbedingte Falten geglättet (z. B. Nasolabialfalte, Mundwinkel), die Enhance-Produkte betonten Gesichtsmerkmale und definierten Konturen neu (Wange, Lippen, Kinn, Nase) und die Skinbooster der Refresh-Serie verbesserten das Hautbild bei jüngeren und älteren Patienten. Die zuvor unter dem Namen Emervel ${ }^{\varpi}$ bekannten Produkte wurden umbenannt und werden nun unter der Dachmarke Restylane ${ }^{\circledast}$ geführt.
Mit Nasolabialfalte und Mundwinkel beginnen

Dr. Said Hilton, Dermatologe aus Düsseldorf, riet in der ersten Sitzung Nasolabialfalte und Mundwinkel zu behandeln, um das Vertrauen des Patienten auch für schwierigere Indikationen zu gewinnen. Bei weiteren Behandlungen im Abstand von etwa einem Monat könnten dann gegebenenfalls Lippen, Wangen, Kieferkontur oder Nasenrücken folgen. Wichtig sei es, zunächst möglichst wenig Material zu verwenden, um ein natürliches Ergebnis zu erreichen - daher arbeite er meist mit kleinen Nadeln. Überzeugt zeigte sich Hilton vom Einsatz von Skinboostern wie Restylane Vital Light für oberflächliche Fältchen, im Bereich des Dekolletés und bei dünner Haut. Zudem seien damit auch Lach- und Knitterfalten an Auge und Wange zu behandeln: „Das klappt nicht mit einem klassischen Filler", so der Ästhetik-Experte. Susanne Pickl

Mittagsseminar „Der Ästhetik-Algorithmus in Theorie und Praxis: Systematische Patientenevaluation und Produktwahl für verbesserte Behandlungsergebnisse" im Rahmen der 25. Fortbildungswoche für praktische Dermatologie und Venerologie, München, 26.7.2016; Veranstalter: Galderma Laboratorium

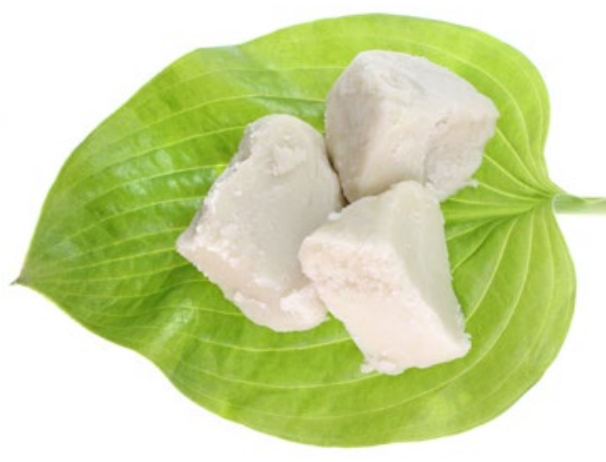

Sheabutter und Hagebutte wirken zusätzlich hautregenerierend.

kung von Milchsäure und Bacillus Ferment beseitigt. Die hautregenerierenden Inhaltsstoffe Allantoin und Panthenol sowie Sheabutter lindern Trockenheit und Risse. Auch die Wirksamkeit ist klinisch belegt: So konnte bei $97 \%$ der Anwendungen nach 14 Tagen ein deutlich besserer Hautzustand an den Fersen festgestellt werden. Die Gel-Oil-Textur ist frisch und leicht, nicht fettend und zieht schnell ein - ideal für trockene Füße.

Neu hinzu kommt jetzt Ureadin ${ }^{\oplus}$ PodosDb, welches speziell für empfindliche diabetische Füße entwickelt wurde. Das dort enthaltene $5 \%$ Urea Isdin ${ }^{\varpi}$ spendet intensive Feuchtigkeit, Polidocanol lindert außerdem den Juckreiz. Ureadin ${ }^{\circledast}$ PodosDb hilft, das Risiko für Ulzerationen zu verringern, verbessert die Mikrozirkulation sowie die Hautelastizität. Dank Hagebutte und BioEcolia hilft es außerdem bei der natürlichen Reparatur der Haut und sorgt künftigen Hautproblemen vor. red

Nach Informationen von ISDIN 